\title{
Local Well-Posedness for the Compressible Nematic Liquid Crystals Flow with Vacuum
}

\author{
Jishan Fan $^{1}$, Fucai Li ${ }^{2, *}$ and Gen Nakamura ${ }^{3}$ \\ ${ }^{1}$ Department of Applied Mathematics, Nanjing Forestry University, Nanjing, \\ Jiangsu 210037, China \\ 2 Department of Mathematics, Nanjing University, Nanjing, Jiangsu 210093, China \\ ${ }^{3}$ Department of Mathematics, Hokkaido University, Sapporo 060-0810, Japan
}

Received 22 August 2019; Accepted (in revised version) 12 January 2020

Dedicated to Professor Weiyi Su on the occasion of her 80th birthday

Abstract. In this paper we prove the local well-posedness of strong solutions to the compressible nematic liquid crystals flow with vacuum in a bounded domain $\Omega \subset \mathbb{R}^{3}$.

Key Words: Liquid crystals, vacuum, Local well-posedness, strong solution.

AMS Subject Classifications: 76D03, 35Q30, 35Q35

\section{Introduction}

In this paper we consider the following simplified version of the Ericksen-Leslie system modeling the flow of compressible nematic liquid crystals (see [2,3]):

$$
\begin{aligned}
& \partial_{t} \rho+\operatorname{div}(\rho u)=0, \\
& \partial_{t}(\rho u)+\operatorname{div}(\rho u \otimes u)+\nabla p(\rho)-\mu \Delta u-(\lambda+\mu) \nabla \operatorname{div} u=-\nabla d \cdot \Delta d, \\
& \partial_{t} d+u \cdot \nabla d-\Delta d=d|\nabla d|^{2} \quad \text { in } \Omega \times(0, T),
\end{aligned}
$$

with boundary and initial conditions:

$$
\begin{aligned}
& u=0, \quad \frac{\partial d}{\partial n}=0 \quad \text { on } \partial \Omega \times(0, T), \\
& (\rho, u, d)(\cdot, 0)=\left(\rho_{0}, u_{0}, d_{0}\right)(\cdot) \quad \text { in } \Omega \subset \mathbb{R}^{3} \text {, }
\end{aligned}
$$

where $\rho \geq 0$ is the density of the fluid, $u \in \mathbb{R}^{3}$ represents velocity field of the fluid, $d \in \mathbb{S}^{2}$ represents the macroscopic average of the nematic liquid crystals orientation field. The

*Corresponding author. Email addresses: fanjishan@njfu.edu.cn (J. S. Fan), fli@nju.edu.cn (F. C. Li), nakamuragenn@gmail.com (G. Nakamura) 
parameters $\mu$ and $\lambda$ are shear viscosity and the bulk viscosity coefficients of the fluid, respectively, satisfying the physical conditions:

$$
\mu>0, \quad 3 \lambda+2 \mu \geq 0 .
$$

We assume that the pressure $p$ satisfies the $\gamma$-law, i.e., $p=: a \rho^{\gamma}$ with constants $a>0$ and $\gamma>1$. The domain $\Omega \subset \mathbb{R}^{3}$ is a bounded domain with smooth boundary $\partial \Omega$, and $n$ is the unit outward normal vector to $\partial \Omega$.

Below let us review some results to the system (1.1a)-(1.1c) briefly. Ding et al. [2] first introduced the system (1.1a)-(1.1c) and studied the low Mach number limit of it, see [9-11] on the recent progress on this topic. Huang, Wang, and Wen [3] (see also [1,5]) showed the local well-posedness of strong solutions with vacuum under the following compatibility condition:

$$
-\mu \Delta u_{0}-(\lambda+\mu) \nabla \operatorname{div} u_{0}+\nabla\left(a \rho_{0}^{\gamma}\right)+\nabla d_{0} \cdot \Delta d_{0}=\sqrt{\rho_{0}} g
$$

for some $g \in L^{2}(\Omega)$. Jiang, Jiang, and Wang [4] (see also [6]) proved the global existence of weak solutions in $\mathbb{R}^{2}$. Lin, Lai and Wang [7] established the existence of global weak solutions with finite energy and density satisfying the renormalized continuity equation, provided the initial orientation director field lies in the hemisphere $\mathbb{S}_{+}^{2}$.

The purpose of this paper is to establish the local well-posedness of strong solutions of the compressible nematic liquid crystal model (1.1a)-(1.1c) without the compatibility condition (1.3).

We will prove

Theorem 1.1. Let $0 \leq \rho_{0} \in W^{1, q},(3<q<6), u_{0} \in H_{0}^{1}, d_{0} \in H^{2}$ with $\left|d_{0}\right|=1$. Then the problem (1.1a)-(1.2b) has a unique local strong solution $(\rho, u, d)$ satisfying

$$
\begin{cases}\rho \in C\left([0, T] ; L^{2}\right) \cap L^{\infty}\left(0, T ; W^{1, q}\right), & \partial_{t} \rho \in L^{\infty}\left(0, T ; L^{2}\right), \\ \rho u \in C\left([0, T] ; L^{2}\right), u \in L^{\infty}\left(0, T ; H_{0}^{1}\right) \cap L^{2}\left(0, T ; H^{2}\right), & \sqrt{\rho} \partial_{t} u \in L^{2}\left(0, T ; L^{2}\right), \\ \sqrt{t} u \in L^{\infty}\left(0, T ; H^{2}\right) \cap L^{2}\left(0, T ; W^{2, q}\right), & \sqrt{t} \partial_{t} u \in L^{2}\left(0, T ; H_{0}^{1}\right), \\ d \in L^{\infty}\left(0, T ; H^{2}\right) \cap L^{2}\left(0, T ; H^{3}\right), \partial_{t} d \in L^{2}\left(0, T ; H^{1}\right), & \sqrt{t} \partial_{t} d \in L^{\infty}\left(0, T ; H^{1}\right),\end{cases}
$$

for some $0<T \leq \infty$.

We will prove Theorem 1.1 in the following way: For $\delta>0$, we choose $0<\delta \leq \rho_{0}^{\delta} \in$ $H^{2}$ and $u_{0}^{\delta} \in H_{0}^{1} \cap H^{2}$ satisfying

$$
\rho_{0}^{\delta} \rightarrow \rho_{0} \quad \text { in } W^{1, q} \quad \text { and } \quad u_{0}^{\delta} \rightarrow u_{0} \quad \text { in } H_{0}^{1} \quad \text { as } \delta \rightarrow 0 .
$$

Then it is easy to verify that the problem (1.1a)-(1.2b) has a unique local strong solution $\left(\rho^{\delta}, u^{\delta}, d^{\delta}\right)$ in $\left[0, T_{\delta}\right)$. 
We further define

$$
\begin{aligned}
M(t)=: 1+ & \sup _{0 \leq s \leq t}\left\{\|\rho(\cdot, s)\|_{W^{1, q}}+\|u(\cdot, s)\|_{H^{1}}+\sqrt{s}\left\|\sqrt{\rho} u_{t}(\cdot, s)\right\|_{L^{2}}\right. \\
& \left.+\|d(\cdot, s)\|_{H^{2}}+\sqrt{s}\left\|d_{t}(\cdot, s)\right\|_{H^{1}}\right\}+\|u\|_{L^{2}\left(0, t ; H^{2}\right)} \\
& +\left\|\sqrt{\rho} u_{t}\right\|_{L^{2}\left(0, t ; L^{2}\right)}+\left\|\sqrt{s} \nabla u_{t}\right\|_{L^{2}\left(0, t ; L^{2}\right)} \\
& +\|d\|_{L^{2}\left(0, t ; H^{3}\right)}+\left\|d_{t}\right\|_{L^{2}\left(0, t ; H^{1}\right)}
\end{aligned}
$$

and prove

Theorem 1.2. For any $t \in\left[0, T_{\delta}\right)$, we have that

$$
M(t) \leq C_{0}\left(M_{0}\right) \exp \left\{t^{\frac{6-q}{4 q}} C(M)\right\}
$$

for some nondecreasing continuous functions $C_{0}(\cdot)$ and $C(\cdot)$.

It follows from (1.7) that (see [8]):

$$
M(t) \leq C
$$

and thus the proof of existence part of Theorem 1.1 is complete by taking $\delta \rightarrow 0$ and the standard compactness principle. We present the proof of Theorem 1.2 in Section 2 and the uniqueness part of Theorem 1.1 in Section 3.

\section{Proof of Theorem 1.2}

Below, for the sake of notational simplicity, we shall drop the superscript " $\delta^{\prime \prime}$ of $\rho^{\delta}, u^{\delta}$ and $d^{\delta}$. We also ignore to write down the domain $\Omega$ in the subsequent integrals.

Testing $(1.1 \mathrm{~b})$ by $u$ and using (1.1a), we see that

$$
\begin{aligned}
& \frac{1}{2} \frac{\mathrm{d}}{\mathrm{d} t} \int \rho|u|^{2} \mathrm{~d} x+\mu \int|\nabla u|^{2} \mathrm{~d} x+(\lambda+\mu) \int(\operatorname{div} u)^{2} \mathrm{~d} x \\
= & \int p \operatorname{div} u \mathrm{~d} x-\int(u \cdot \nabla) d \cdot \Delta d \mathrm{~d} x .
\end{aligned}
$$

Testing (1.1c) by $-\Delta d-d|\nabla d|^{2}$ and using $d \cdot d_{t}=0$ and $d \cdot \nabla d=0$, we find that

$$
\frac{1}{2} \frac{\mathrm{d}}{\mathrm{d} t} \int|\nabla d|^{2} \mathrm{~d} x+\left.\left.\int|\Delta d+d| \nabla d\right|^{2}\right|^{2} \mathrm{~d} x=\int(u \cdot \nabla) d \cdot \Delta d \mathrm{~d} x .
$$

Summing (2.1) and (2.2) up, and rewriting the continuity equation (1.1a) as

$$
p_{t}+u \cdot \nabla p+\gamma p \operatorname{div} u=0
$$


we infer that

$$
\begin{aligned}
& \frac{1}{2} \frac{\mathrm{d}}{\mathrm{d} t} \int\left(\rho|u|^{2}+|\nabla d|^{2}\right) \mathrm{d} x+\int\left(\mu|\nabla u|^{2}+(\lambda+\mu)(\operatorname{div} u)^{2}+\left.\left.|\Delta d+d| \nabla d\right|^{2}\right|^{2}\right) \mathrm{d} x \\
= & \int p \operatorname{div} u \mathrm{~d} x=-\frac{1}{\gamma-1} \int p_{t} \mathrm{~d} x,
\end{aligned}
$$

which gives

$$
\begin{aligned}
& \int\left(\rho|u|^{2}+|\nabla d|^{2}+\frac{2 a}{\gamma-1} \rho^{\gamma}\right) \mathrm{d} x+2 \int_{0}^{T} \int\left(|\nabla u|^{2}+\left.\left.|\Delta d+d| \nabla d\right|^{2}\right|^{2}\right) \mathrm{d} x \mathrm{~d} t \\
\leq & \int\left(\rho_{0}\left|u_{0}\right|^{2}+\left|\nabla d_{0}\right|^{2}+\frac{2 a}{\gamma-1} \rho_{0}^{\gamma}\right) \mathrm{d} x .
\end{aligned}
$$

Testing (1.1c) by $d_{t}$ and using $d \cdot d_{t}=0$, we find that

$$
\begin{aligned}
& \frac{1}{2} \frac{\mathrm{d}}{\mathrm{d} t} \int|\nabla d|^{2} \mathrm{~d} x+\int\left|d_{t}\right|^{2} \mathrm{~d} x=-\int u \cdot \nabla d \cdot d_{t} \mathrm{~d} x \\
\leq & \|u\|_{L^{6}}\|\nabla d\|_{L^{3}}\left\|d_{t}\right\|_{L^{2}} \leq C(M)\left\|d_{t}\right\|_{L^{2}} \\
\leq & \frac{1}{2}\left\|d_{t}\right\|_{L^{2}}^{2}+C(M),
\end{aligned}
$$

which gives

$$
\int_{0}^{t}\left\|d_{t}\right\|_{L^{2}}^{2} \mathrm{~d} s \leq C_{0}\left(M_{0}\right)+C(M) t .
$$

Applying $\nabla$ to (1.1c), we see that

$$
\nabla d_{t}-\nabla \Delta d+\nabla(u \cdot \nabla d)=\nabla\left(d|\nabla d|^{2}\right)
$$

Testing (2.6) by $\nabla d_{t}$, we deduce that

$$
\begin{aligned}
& \frac{1}{2} \frac{\mathrm{d}}{\mathrm{d} t} \int|\Delta d|^{2} \mathrm{~d} x+\int\left|\nabla d_{t}\right|^{2} \mathrm{~d} x \\
= & \int\left(\nabla\left(d|\nabla d|^{2}\right)-\nabla(u \cdot \nabla d)\right) \nabla d_{t} \mathrm{~d} x \\
\leq & C\left(\|\nabla d\|_{L^{6}}^{3}+\|\nabla d\|_{L^{\infty}}\left\|\nabla^{2} d\right\|_{L^{2}}^{2}+\|\nabla u\|_{L^{2}}\|\nabla d\|_{L^{\infty}}+\|u\|_{L^{6}}\left\|\nabla^{2} d\right\|_{L^{3}}\right)\left\|\nabla d_{t}\right\|_{L^{2}} \\
\leq & C(M)\left(1+\|\nabla d\|_{L^{\infty}}+\left\|\nabla^{2} d\right\|_{L^{3}}\right)\left\|\nabla d_{t}\right\|_{L^{2}} \\
\leq & C(M)\left(1+\|d\|_{H^{3}}^{\frac{1}{2}}\right)\left\|\nabla d_{t}\right\|_{L^{2}} \\
\leq & \frac{1}{2}\left\|\nabla d_{t}\right\|_{L^{2}}^{2}+C(M)+C(M)\|d\|_{H^{3}}
\end{aligned}
$$

which leads to

$$
\int|\Delta d|^{2} \mathrm{~d} x+\int_{0}^{t}\left\|\nabla d_{t}\right\|_{L^{2}}^{2} \mathrm{~d} s \leq C_{0}\left(M_{0}\right)+C(M) t^{\frac{1}{2}}
$$


Here we have used the Gagliardo-Nirenberg inequalities

$$
\begin{aligned}
& \|\nabla d\|_{L^{\infty}}^{2} \leq C\left\|\nabla^{2} d\right\|_{L^{2}}\|d\|_{H^{3}} \\
& \left\|\nabla^{2} d\right\|_{L^{3}}^{2} \leq C\left\|\nabla^{2} d\right\|_{L^{2}}\|d\|_{H^{3}} .
\end{aligned}
$$

Eq. (1.1b) can be written as

$$
-\mu \Delta u-(\lambda+\mu) \nabla \operatorname{div} u=f=:-\rho \partial_{t} u-\rho u \cdot \nabla u-\nabla p(\rho)-\nabla d \cdot \Delta d .
$$

Then we have

$$
\begin{aligned}
\|u\|_{W^{2, q}} & \leq C\|f\|_{L^{q}} \leq C\left\|\rho \partial_{t} u\right\|_{L^{q}}+C\|\rho u \cdot \nabla u\|_{L^{q}}+C\|\nabla p\|_{L^{q}}+C\|\nabla d\|_{L^{\infty}}\|\Delta d\|_{L^{q}} \\
& \leq C\|\rho\|_{L^{\infty}}^{\frac{5 q-6}{4 q}}\left\|\sqrt{\rho} u_{t}\right\|_{L^{2}}^{\frac{6-q}{2 q}}\left\|u_{t}\right\|_{L^{6}}^{\frac{3 q-6}{2 q}}+C(M)\|u\|_{L^{\infty}}\|\nabla u\|_{L^{q}}+C(M)\|d\|_{H^{3}}^{\frac{3}{2}} \\
& \leq C(M)\left\|\sqrt{\rho} u_{t}\right\|_{L^{2}}^{\frac{6-q}{2 q}}\left\|\nabla u_{t}\right\|_{L^{2}}^{\frac{3 q-6}{2 q}}+C(M)\|\nabla u\|_{L^{2}}^{\frac{1}{2}}\|u\|_{H^{2}}^{\frac{3}{2}}+C(M)\|d\|_{H^{3}}^{\frac{3}{2}} \\
& \leq C(M)\left\|\sqrt{\rho} u_{t}\right\|_{L^{2}}^{\frac{6-q}{2 q}}\left\|\nabla u_{t}\right\|_{L^{2}}^{\frac{3 q-6}{2 q}}+C(M)\|u\|_{H^{2}}^{\frac{3}{2}}+C(M)\|d\|_{H^{3}}^{\frac{3}{2}},
\end{aligned}
$$

which gives

$$
\begin{aligned}
& \int_{0}^{t}\|u\|_{W^{2, q}} \mathrm{~d} s \leq C(M) \int_{0}^{t}\left\|\sqrt{\rho} u_{t}\right\|_{L^{2}}^{\frac{6-q}{2 q}}\left\|\nabla u_{t}\right\|_{L^{2}}^{\frac{3 q-6}{2 q}} \mathrm{~d} s+C(M) \int_{0}^{t}\|u\|_{H^{2}}^{\frac{3}{2}} \mathrm{~d} s+C(M) \int_{0}^{t}\|d\|_{H^{3}}^{\frac{3}{2}} \mathrm{~d} s \\
& \leq C(M) \int_{0}^{t} s^{-\frac{3 q-6}{4 q}}\left(\sqrt{s}\left\|\nabla u_{t}\right\|_{L^{2}}\right)^{\frac{3 q-6}{2 q}}\left\|\sqrt{\rho} u_{t}\right\|_{L^{2}}^{\frac{6-q}{2 q}} \mathrm{~d} s \\
& \quad+C(M)\left(\int_{0}^{t} \mathrm{~d} s\right)^{\frac{1}{4}}\left(\int_{0}^{t}\|u\|_{H^{2}}^{2} \mathrm{~d} s\right)^{\frac{3}{4}}+C(M)\left(\int_{0}^{t} \mathrm{~d} s\right)^{\frac{1}{4}}\left(\int_{0}^{t}\|d\|_{H^{3}}^{2} \mathrm{~d} s\right)^{\frac{3}{4}} \\
& \leq C(M)\left(\int_{0}^{t} s^{-\frac{3 q-6}{2 q}} \mathrm{~d} s\right)^{\frac{1}{2}}\left(\int_{0}^{t} s\left\|\nabla u_{t}\right\|_{L^{2}}^{2} \mathrm{~d} s\right)^{\frac{3 q-6}{4 q}}\left(\int_{0}^{t}\left\|\sqrt{\rho} u_{t}\right\|_{L^{2}}^{2} \mathrm{~d} s\right)^{\frac{6-q}{4 q}}+C(M) t^{\frac{1}{4}} \\
& \leq C(M) t^{\frac{6-q}{4 q}}+C(M) t^{\frac{1}{4}} \leq C(M) t^{\frac{6-q}{4 q}}
\end{aligned}
$$

for all $0<t \leq 1$.

Using the Gagliardo-Nirenberg inequality

$$
\|\nabla u\|_{L^{\infty}} \leq C\|\nabla u\|_{L^{2}}^{\frac{2 q-6}{5 q-6}}\|u\|_{W^{2, q}}^{\frac{3 q}{5-6}}
$$

we observe that

$$
\begin{aligned}
& \int_{0}^{t}\|\nabla u\|_{L^{\infty}} \mathrm{d} s \leq C(M) \int_{0}^{t}\|u\|_{W^{2, q}}^{\frac{3 q}{5 q-6}} \mathrm{~d} s \\
\leq & C\left(\int_{0}^{t} \mathrm{~d} s\right)^{\frac{2 q-6}{5 q-6}}\left(\int_{0}^{t}\|u\|_{W^{2, q}} \mathrm{~d} s\right)^{\frac{3 q}{5 q-6}} \\
\leq & C(M) t^{\frac{2 q-6}{5 q-6}} \cdot t^{\frac{6-q}{4 q} \cdot \frac{3 q}{5 q-6}}=C(M) t^{\frac{6-q}{4 q}} .
\end{aligned}
$$


Testing (1.1a) by $\rho^{m-1}$, we see that

$$
\frac{1}{m} \frac{\mathrm{d}}{\mathrm{d} t} \int \rho^{m} \mathrm{~d} x=-\int \operatorname{div}(\rho u) \rho^{m-1} \mathrm{~d} x=\int \rho u \nabla \rho^{m-1} \mathrm{~d} x=-\frac{m-1}{m} \int \rho^{m} \operatorname{div} u \mathrm{~d} x,
$$

which leads to

$$
\frac{\mathrm{d}}{\mathrm{d} t}\|\rho\|_{L^{m}} \leq\|\operatorname{div} u\|_{L^{\infty}}\|\rho\|_{L^{m}}
$$

and thus

$$
\begin{aligned}
\|\rho\|_{L^{m}} & \leq\left\|\rho_{0}\right\|_{L^{m}} \exp \left(\int_{0}^{t}\|\operatorname{div} u\|_{L^{\infty}} \mathrm{d} s\right) \\
& \leq\left\|\rho_{0}\right\|_{L^{m}} \exp \left\{t^{\frac{6-q}{4 q}} C(M)\right\}, \quad 2 \leq m<\infty .
\end{aligned}
$$

For $m=\infty$, (2.13) still holds.

Taking $\nabla$ to (1.1a), testing the result by $|\nabla \rho|^{q-2} \nabla \rho$, we find that

$$
\frac{\mathrm{d}}{\mathrm{d} t}\|\nabla \rho\|_{L^{q}} \leq C\|\nabla u\|_{L^{\infty}}\|\nabla \rho\|_{L^{q}}+C\|\rho\|_{L^{\infty}}\|\nabla \operatorname{div} u\|_{L^{q}},
$$

which implies

$$
\begin{aligned}
\|\nabla \rho\|_{L^{q}} & \leq C\left(\left\|\nabla \rho_{0}\right\|_{L^{q}}+\int_{0}^{t}\|\rho\|_{L^{\infty}}\|\nabla \operatorname{div} u\|_{L^{q}} \mathrm{~d} s\right) \exp \left(\int_{0}^{t}\|\nabla u\|_{L^{\infty}} \mathrm{d} s\right) \\
& \leq C\left(1+C(M) t^{\frac{6-q}{4 q}}\right) \exp \left\{t^{\frac{6-q}{4 q}} C(M)\right\} \\
& \leq C_{0}\left(M_{0}\right) \exp \left\{t^{\frac{6-q}{4 q}} C(M)\right\} .
\end{aligned}
$$

Testing (1.1b) by $u_{t}$, we deduce that

$$
\begin{aligned}
& \frac{1}{2} \frac{\mathrm{d}}{\mathrm{d} t} \int\left(\mu|\nabla u|^{2}+(\lambda+\mu)(\operatorname{div} u)^{2}\right) \mathrm{d} x+\int \rho\left|u_{t}\right|^{2} \mathrm{~d} x \\
= & -\int \rho u \cdot \nabla u \cdot u_{t} \mathrm{~d} x+\int p \operatorname{div} u_{t} \mathrm{~d} x+\int\left(\nabla d \odot \nabla d-\frac{1}{2}|\nabla d|^{2} \mathbb{I}_{3}\right): \nabla u_{t} \mathrm{~d} x \\
= & : I_{1}+I_{2}+I_{3} .
\end{aligned}
$$

We bound $I_{1}, I_{2}$ and $I_{3}$ as follows.

$$
\begin{aligned}
\left|I_{1}\right| & \leq\left\|\sqrt{\rho} u_{t}\right\|_{L^{2}}\|\sqrt{\rho}\|_{L^{\infty}}\|u\|_{L^{6}}\|\nabla u\|_{L^{3}} \\
& \leq C(M)\left\|\sqrt{\rho} u_{t}\right\|_{L^{2}}\|\nabla u\|_{L^{2}}^{\frac{1}{2}}\|u\|_{H^{2}}^{\frac{1}{2}} \\
& \leq C(M)\left\|\sqrt{\rho} u_{t}\right\|_{L^{2}}\|u\|_{H^{2}}^{\frac{1}{2}} \\
& \leq \frac{1}{4}\left\|\sqrt{\rho} u_{t}\right\|_{L^{2}}^{2}+C(M)\|u\|_{H^{2}}
\end{aligned}
$$




$$
\begin{aligned}
I_{2} & =\frac{\mathrm{d}}{\mathrm{d} t} \int p \operatorname{div} u \mathrm{~d} x-\int p_{t} \operatorname{div} u \mathrm{~d} x \\
& =\frac{\mathrm{d}}{\mathrm{d} t} \int p \operatorname{div} u \mathrm{~d} x+\int(u \cdot \nabla p+\gamma p \operatorname{div} u) \operatorname{div} u \mathrm{~d} x \\
& \leq \frac{\mathrm{d}}{\mathrm{d} t} \int p \operatorname{div} u \mathrm{~d} x+\left(\|u\|_{L^{6}}\|\nabla p\|_{L^{3}}+\gamma\|p\|_{L^{\infty}}\|\operatorname{div} u\|_{L^{2}}\right)\|\operatorname{div} u\|_{L^{2}} \\
& \leq \frac{\mathrm{d}}{\mathrm{d} t} \int p \operatorname{div} u \mathrm{~d} x+C(M) .
\end{aligned}
$$

Here we have used the equality (2.3). And for the term $I_{3}$, it holds

$$
\begin{aligned}
I_{3} & =\frac{\mathrm{d}}{\mathrm{d} t} \int\left(\nabla d \odot \nabla d-\frac{1}{2}|\nabla d|^{2} \mathbb{I}_{3}\right): \nabla u \mathrm{~d} x-\int \partial_{t}\left(\nabla d \odot \nabla d-\frac{1}{2}|\nabla d|^{2} \mathbb{I}_{3}\right): \nabla u \mathrm{~d} x \\
& \leq \frac{\mathrm{d}}{\mathrm{d} t} \int\left(\nabla d \odot \nabla d-\frac{1}{2}|\nabla d|^{2} \mathbb{I}_{3}\right): \nabla u \mathrm{~d} x+C\|\nabla d\|_{L^{\infty}}\left\|\nabla d_{t}\right\|_{L^{2}}\|\nabla u\|_{L^{2}} \\
& \leq \frac{\mathrm{d}}{\mathrm{d} t} \int\left(\nabla d \odot \nabla d-\frac{1}{2}|\nabla d|^{2} \mathbb{I}_{3}\right): \nabla u \mathrm{~d} x+C(M)\|\nabla d\|_{L^{\infty}}\left\|\nabla d_{t}\right\|_{L^{2}} .
\end{aligned}
$$

Inserting the above estimates into (2.15), integrating over $(0, t)$, and using (2.7), we have

$$
\begin{aligned}
& \|\nabla u\|_{L^{2}}^{2}+\int_{0}^{t}\left\|\sqrt{\rho} u_{t}\right\|_{L^{2}}^{2} \mathrm{~d} s \\
\leq & C_{0}\left(M_{0}\right)+C(M) t^{\frac{1}{2}}+C(M) t+C\|\nabla d\|_{L^{4}}^{2} \\
\leq & C_{0}\left(M_{0}\right)+C(M) t^{\frac{1}{2}}+C\left\|\nabla^{2} d\right\|_{L^{2}} \\
\leq & C_{0}\left(M_{0}\right)+C(M) t^{\frac{1}{2}} \leq C_{0}\left(M_{0}\right) \exp \left\{t^{\frac{6-q}{4 q}} C(M)\right\} .
\end{aligned}
$$

Applying $\partial_{t}$ to $(1.1 \mathrm{~b})$ and using (1.1a), we infer that

$$
\begin{gathered}
\rho \partial_{t}^{2} u+\rho u \cdot \nabla u_{t}-\mu \Delta u_{t}-(\lambda+\mu) \nabla \operatorname{div} u_{t} \\
=-\nabla p_{t}+\operatorname{div}(\rho u)\left(u_{t}+u \cdot \nabla u\right)-\rho u_{t} \cdot \nabla u \\
-\partial_{t} \operatorname{div}\left(\nabla d \odot \nabla d-\frac{1}{2}|\nabla d|^{2} \mathbb{I}_{3}\right) .
\end{gathered}
$$

Testing (2.23) by $u_{t}$ and using (1.1a), we have

$$
\begin{aligned}
& \frac{1}{2} \frac{\mathrm{d}}{\mathrm{d} t} \int \rho\left|u_{t}\right|^{2} \mathrm{~d} x+\int\left(\mu\left|\nabla u_{t}\right|^{2}+(\lambda+\mu)\left(\operatorname{div} u_{t}\right)^{2}\right) \mathrm{d} x \\
= & \int p_{t} \operatorname{div} u_{t} \mathrm{~d} x-\int \rho u \nabla\left|u_{t}\right|^{2} \mathrm{~d} x-\int \rho u \cdot \nabla\left(u \cdot \nabla u \cdot u_{t}\right) \mathrm{d} x \\
& \quad-\int \rho u_{t} \cdot \nabla u \cdot u_{t} \mathrm{~d} x+\int \partial_{t}\left(\nabla d \odot \nabla d-\frac{1}{2}|\nabla d|^{2} \mathbb{I}_{3}\right): \nabla u_{t} \mathrm{~d} x \\
= & : \sum_{i=4}^{8} I_{i} .
\end{aligned}
$$


We bound $I_{i}(i=4, \cdots, 8)$ as follows.

$$
\begin{aligned}
\left|I_{4}\right| & =\left|\int(u \cdot \nabla p+\gamma p \operatorname{div} u) \operatorname{div} u_{t} \mathrm{~d} x\right| \\
\leq & \left(\|u\|_{L^{6}}\|\nabla p\|_{L^{3}}+\gamma\|p\|_{L^{\infty}}\|\operatorname{div} u\|_{L^{2}}\right)\left\|\operatorname{div} u_{t}\right\|_{L^{2}} \\
\leq & C(M)\left\|\nabla u_{t}\right\|_{L^{2}} \leq \frac{\mu}{16}\left\|\nabla u_{t}\right\|_{L^{2}}^{2}+C(M) \\
\left|I_{5}\right| \leq & \|\sqrt{\rho}\|_{L^{\infty}}\left\|\sqrt{\rho} u_{t}\right\|_{L^{3}}\|u\|_{L^{6}}\left\|\nabla u_{t}\right\|_{L^{2}} \leq C(M)\left\|\sqrt{\rho} u_{t}\right\|_{L^{3}}\left\|\nabla u_{t}\right\|_{L^{2}} \\
\leq & C(M)\left\|\sqrt{\rho} u_{t}\right\|_{L^{2}}^{\frac{1}{2}}\left\|\sqrt{\rho} u_{t}\right\|_{L^{6}}^{\frac{1}{2}}\left\|\nabla u_{t}\right\|_{L^{2}} \leq C(M)\left\|\sqrt{\rho} u_{t}\right\|_{L^{2}}^{\frac{1}{2}}\left\|\nabla u_{t}\right\|_{L^{2}}^{\frac{3}{2}} \\
\leq & \frac{\mu}{16}\left\|\nabla u_{t}\right\|_{L^{2}}^{2}+C(M)\left\|\sqrt{\rho} u_{t}\right\|_{L^{2}}^{2} \\
\left|I_{6}\right| \leq & \|\rho\|_{L^{\infty}}\|u\|_{L^{6}}\|\nabla u\|_{L^{3}}^{2}\left\|u_{t}\right\|_{L^{6}}+\|\rho\|_{L^{\infty}}\|u\|_{L^{6}}^{2}\left\|\nabla^{2} u\right\|_{L^{2}}\left\|u_{t}\right\|_{L^{6}} \\
& +\|\rho\|_{L^{\infty}}\|u\|_{L^{6}}^{2}\|\nabla u\|_{L^{6}}\left\|\nabla u_{t}\right\|_{L^{2}} \\
\leq & C(M)\left(\|\nabla u\|_{L^{3}}^{2}+\|u\|_{H^{2}}\right)\left\|\nabla u_{t}\right\|_{L^{2}} \\
\leq & C(M)\left(\|\nabla u\|_{L^{2}}\|u\|_{H^{2}}+\|u\|_{H^{2}}\right)\left\|\nabla u_{t}\right\|_{L^{2}} \\
\leq & C(M)\|u\|_{H^{2}}\left\|\nabla u_{t}\right\|_{L^{2}} \leq \frac{\mu}{16}\left\|\nabla u_{t}\right\|_{L^{2}}^{2}+C(M)\|u\|_{H^{2}}^{2}, \\
\left|I_{7}\right| \leq & \|\nabla u\|_{L^{2}}\left\|\sqrt{\rho} u_{t}\right\|_{L^{4}}^{2} \leq\|\nabla u\|_{L^{2}}\left\|\sqrt{\rho} u_{t}\right\|_{L^{2}}^{\frac{1}{2}}\left\|\sqrt{\rho} u_{t}\right\|_{L^{6}}^{\frac{3}{2}} \\
\leq & C(M)\left\|\sqrt{\rho} u_{t}\right\|_{L^{2}}^{\frac{1}{2}}\left\|\nabla u_{t}\right\|_{L^{2}}^{\frac{3}{2}} \leq \frac{\mu}{16}\left\|\nabla u_{t}\right\|_{L^{2}}^{2}+C(M)\left\|\sqrt{\rho} u_{t}\right\|_{L^{2}}^{2} \\
\left|I_{8}\right| \leq & C\left\|\nabla u_{t}\right\|_{L^{2}}\|\nabla d\|_{L^{\infty}}\left\|\nabla d_{t}\right\|_{L^{2}} \frac{\mu}{16}\left\|\nabla u_{t}\right\|_{L^{2}}^{2}+C\|\nabla d\|_{L^{\infty}}^{2}\left\|\nabla d_{t}\right\|_{L^{2}}^{2} \\
\leq & \frac{\mu}{16}\left\|\nabla u_{t}\right\|_{L^{2}}^{2}+C(M)\|d\|_{H^{3}}\left\|\nabla d_{t}\right\|_{L^{2}}^{2} .
\end{aligned}
$$

Inserting the above estimates into (2.18) gives

$$
\begin{aligned}
& \frac{1}{2} \frac{\mathrm{d}}{\mathrm{d} t} \int \rho\left|u_{t}\right|^{2} \mathrm{~d} x+\frac{11}{16} \mu \int\left|\nabla u_{t}\right|^{2} \mathrm{~d} x \\
\leq & C(M)+C(M)\left\|\sqrt{\rho} u_{t}\right\|_{L^{2}}^{2}+C(M)\|u\|_{H^{2}}^{2}+C(M)\|d\|_{H^{3}}\left\|\nabla d_{t}\right\|_{L^{2}}^{2} .
\end{aligned}
$$

Multiplying the above inequality by $t$, we obtain

$$
\begin{aligned}
& \quad \frac{1}{2} \frac{\mathrm{d}}{\mathrm{d} t}\left(t \int \rho u_{t}^{2} \mathrm{~d} x\right)+\frac{11}{16} \mu t \int\left|\nabla u_{t}\right|^{2} \mathrm{~d} x \\
& \leq \frac{1}{2} \int \rho\left|u_{t}\right|^{2} \mathrm{~d} x+C(M) t+C(M) t \int \rho\left|u_{t}\right|^{2} \mathrm{~d} x+C(M) t\|u\|_{H^{2}}^{2} \\
& \quad+C(M)\|d\|_{H^{3}} \cdot t\left\|\nabla d_{t}\right\|_{L^{2}}^{2}
\end{aligned}
$$

which implies that

$$
t \int \rho\left|u_{t}\right|^{2} \mathrm{~d} x+\int_{0}^{t} s\left\|\nabla u_{t}\right\|_{L^{2}}^{2} \mathrm{~d} s \leq C_{0}\left(M_{0}\right) \exp \left\{t^{\frac{6-q}{4 q}} C(M)\right\} .
$$


It follows from (2.9) that

$$
\begin{aligned}
\|u\|_{H^{2}} \leq C\|f\|_{L^{2}} \leq C\left\|\rho u_{t}+\rho u \cdot \nabla u+\nabla p+\nabla d \cdot \Delta d\right\|_{L^{2}} \\
\leq C\|\sqrt{\rho}\|_{L^{\infty}}\left\|\sqrt{\rho} u_{t}\right\|_{L^{2}}+C\|\rho\|_{L^{\infty}}\|u\|_{L^{6}}\|\nabla u\|_{L^{3}} \\
\quad+C\|\nabla p\|_{L^{2}}+C\|\nabla d\|_{L^{\infty}}\|\Delta d\|_{L^{2}} \\
\leq C\|\sqrt{\rho}\|_{L^{\infty}}\left\|\sqrt{\rho} u_{t}\right\|_{L^{2}}+C(M)\|\nabla u\|_{L^{3}}+C(M)+C(M)\|\nabla d\|_{L^{\infty}} \\
\leq C\|\sqrt{\rho}\|_{L^{\infty}}\left\|\sqrt{\rho} u_{t}\right\|_{L^{2}}+C(M)\|\nabla u\|_{L^{2}}^{\frac{1}{2}} \cdot\|u\|_{H^{2}}^{\frac{1}{2}}+C(M)+C(M)\|\nabla d\|_{L^{\infty}},
\end{aligned}
$$

which yields

$$
\|u\|_{H^{2}} \leq C(M)+C\|\sqrt{\rho}\|_{L^{\infty}}\left\|\sqrt{\rho} u_{t}\right\|_{L^{2}}+C(M)\|\nabla d\|_{L^{\infty}},
$$

whence

$$
\|u\|_{L^{2}\left(0, t ; H^{2}\right)} \leq C_{0}\left(M_{0}\right) \exp \left\{t^{\frac{6-q}{4 q}} C(M)\right\} .
$$

Taking $\partial_{t}$ to (1.1c), testing the result by $d_{t}$, and using $d \cdot d_{t}=0$, we observe that

$$
\begin{aligned}
& \frac{1}{2} \frac{\mathrm{d}}{\mathrm{d} t} \int\left|d_{t}\right|^{2} \mathrm{~d} x+\int\left|\nabla d_{t}\right|^{2} \mathrm{~d} x \\
= & -\int u_{t} \cdot \nabla d \cdot d_{t} \mathrm{~d} x-\int u \cdot \nabla d_{t} \cdot d_{t} \mathrm{~d} x+\int\left|d_{t}\right|^{2}|\nabla d|^{2} \mathrm{~d} x \\
\leq & \left\|u_{t}\right\|_{L^{6}}\|\nabla d\|_{L^{3}}\left\|d_{t}\right\|_{L^{2}}+\|u\|_{L^{6}}\left\|\nabla d_{t}\right\|_{L^{2}}\left\|d_{t}\right\|_{L^{3}}+\|\nabla d\|_{L^{6}}^{2}\left\|d_{t}\right\|_{L^{6}}\left\|d_{t}\right\|_{L^{2}} \\
\leq & C\|\nabla d\|_{L^{3}}\left\|\nabla u_{t}\right\|_{L^{2}}\left\|d_{t}\right\|_{L^{2}}+C(M)\left\|\nabla d_{t}\right\|_{L^{2}}\left\|d_{t}\right\|_{L^{3}}+C(M)\left\|d_{t}\right\|_{L^{6}}\left\|d_{t}\right\|_{L^{2}} \\
\leq & \frac{1}{2}\left\|\nabla d_{t}\right\|_{L^{2}}^{2}+C\|\nabla d\|_{L^{3}}\left\|\nabla u_{t}\right\|_{L^{2}}\left\|d_{t}\right\|_{L^{2}}+C(M)\left\|d_{t}\right\|_{L^{2}}^{2}
\end{aligned}
$$

which gives

$$
\frac{1}{2} \frac{\mathrm{d}}{\mathrm{d} t} \int\left|d_{t}\right|^{2} \mathrm{~d} x+\frac{1}{2} \int\left|\nabla d_{t}\right|^{2} \mathrm{~d} x \leq C\|\nabla d\|_{L^{3}}\left\|\nabla u_{t}\right\|_{L^{2}}\left\|d_{t}\right\|_{L^{2}}+C(M)\left\|d_{t}\right\|_{L^{2}}^{2} .
$$

Multiplying the above inequality by $t$, using (2.21), (2.5), and (2.7), we obtain

$$
t \int\left|d_{t}\right|^{2} \mathrm{~d} x+\int_{0}^{t} s\left\|\nabla d_{t}\right\|_{L^{2}}^{2} \mathrm{~d} s \leq C_{0}\left(M_{0}\right) \exp \left\{t^{\frac{6-q}{4 q}} C(M)\right\} .
$$

Taking $\partial_{t}$ to (1.1c), testing the result by $-\Delta d_{t}$, and using (2.5) and (2.7), we have

$$
\begin{aligned}
& \quad \frac{1}{2} \frac{\mathrm{d}}{\mathrm{d} t} \int\left|\nabla d_{t}\right|^{2} \mathrm{~d} x+\int\left|\Delta d_{t}\right|^{2} \mathrm{~d} x \\
& =\int\left(u_{t} \cdot \nabla d+u \cdot \nabla d_{t}\right) \Delta d_{t} \mathrm{~d} x-\int\left(d_{t}|\nabla d|^{2}+d \partial_{t}|\nabla d|^{2}\right) \Delta d_{t} \mathrm{~d} x \\
& \leq\left(\left\|u_{t}\right\|_{L^{6}}\|\nabla d\|_{L^{3}}+\|u\|_{L^{6}}\left\|\nabla d_{t}\right\|_{L^{3}}\right)\left\|\Delta d_{t}\right\|_{L^{2}} \\
& \quad+\left(\left\|d_{t}\right\|_{L^{6}}\|\nabla d\|_{L^{6}}^{2}+\|\nabla d\|_{L^{6}}\left\|\nabla d_{t}\right\|_{L^{3}}\right)\left\|\Delta d_{t}\right\|_{L^{2}} \\
& \leq \\
& \leq \frac{1}{2}\left\|\Delta d_{t}\right\|_{L^{2}}^{2}+C\|\nabla d\|_{L^{3}}^{2}\left\|\nabla u_{t}\right\|_{L^{2}}^{2}+C(M)\left(\left\|\nabla d_{t}\right\|_{L^{2}}^{2}+\left\|d_{t}\right\|_{L^{2}}^{2}\right) .
\end{aligned}
$$


Multiplying the above inequality by $t$, using (2.21), (2.5), (2.7), and (2.23), we have

$$
t \int\left|\nabla d_{t}\right|^{2} \mathrm{~d} x \leq C_{0}\left(M_{0}\right) \exp \left\{t^{\frac{6-q}{4 q}} C(M)\right\} .
$$

It follows from (1.1c) and (2.7) that

$$
\begin{aligned}
&\|d\|_{H^{3}} \leq C\|d\|_{H^{1}}+\|\nabla \Delta d\|_{L^{2}} \\
& \leq C\|d\|_{H^{1}}+\left\|\nabla\left(d_{t}+u \cdot \nabla d-d|\nabla d|^{2}\right)\right\|_{L^{2}} \\
& \leq C+C\left\|\nabla d_{t}\right\|_{L^{2}}+C\|u\|_{L^{6}}\left\|\nabla^{2} d\right\|_{L^{3}}+C\|\nabla u\|_{L^{2}}\|\nabla d\|_{L^{\infty}} \\
& \quad+C\|\nabla d\|_{L^{6}}^{3}+C\|\nabla d\|_{L^{\infty}}\left\|\nabla^{2} d\right\|_{L^{2}} \\
& \quad \leq C+C\left\|\nabla d_{t}\right\|_{L^{2}}+C(M)\|d\|_{H^{3}}^{\frac{1}{2}}+C(M)
\end{aligned}
$$

which implies

$$
\|d\|_{H^{3}} \leq C+C(M)+C\left\|\nabla d_{t}\right\|_{L^{2}}
$$

whence

$$
\begin{aligned}
\|d\|_{L^{2}\left(0, t ; H^{3}\right)} & \leq C t+C(M) t+C(M) t^{\frac{1}{2}} \leq C(M) t^{\frac{1}{2}} \\
& \leq C_{0}\left(M_{0}\right) \exp \left\{t^{\frac{6-q}{4 q}} C(M)\right\} .
\end{aligned}
$$

Combining (2.16) and (2.21)-(2.25), we conclude that (1.7) holds true. This completes the proof of Theorem 1.2.

\section{Proof of Theorem 1.1}

This section is devoted to the proof of Theorem 1.1. Since the existence part has been given, we only need to show the uniqueness part. Let $\left(\rho_{i}, u_{i}, d_{i}\right),(i=1,2)$ be the two strong solutions satisfying (1.4) with the same initial data.

We denote

$$
(\rho, u, d)=:\left(\rho_{1}-\rho_{2}, u_{1}-u_{2}, d_{1}-d_{2}\right) .
$$

Then it is easy to verify that

$$
\begin{aligned}
& \partial_{t} \rho+u_{2} \cdot \nabla \rho+\rho \operatorname{div} u_{2}+\rho_{1} \operatorname{div} u+u \cdot \nabla \rho_{1}=0, \\
& \rho_{1} \partial_{t} u+\rho_{1} u_{1} \cdot \nabla u-\mu \Delta u-(\lambda+\mu) \nabla \operatorname{div} u \\
& =-\rho_{1} u \cdot \nabla u_{2}-\rho\left(\partial_{t} u_{2}+u_{2} \cdot \nabla u_{2}\right)-\nabla\left(p\left(\rho_{1}\right)-p\left(\rho_{2}\right)\right) \\
& \quad-\operatorname{div}\left(\nabla d_{1} \odot \nabla d_{1}-\nabla d_{2} \odot \nabla d_{2}-\frac{1}{2}\left|\nabla d_{1}\right|^{2} \mathbb{I}_{3}+\frac{1}{2}\left|\nabla d_{2}\right|^{2} \mathbb{I}_{3}\right), \\
& \partial_{t} d+u_{1} \nabla d+u \cdot \nabla d_{2}-\Delta d=d_{1}\left|\nabla d_{1}\right|^{2}-d_{2}\left|\nabla d_{2}\right|^{2} .
\end{aligned}
$$


Testing (3.1) by $\rho$ and using (1.4), we have

$$
\begin{aligned}
\frac{1}{2} \frac{\mathrm{d}}{\mathrm{d} t} \int \rho^{2} \mathrm{~d} x & =-\int\left(u_{2} \nabla \rho+\rho \operatorname{div} u_{2}+\rho_{1} \operatorname{div} u+u \cdot \nabla \rho_{1}\right) \rho \mathrm{d} x \\
& =-\int\left(\frac{1}{2} \rho^{2} \operatorname{div} u_{2}+\rho_{1} \operatorname{div} u \rho+u \nabla \rho_{1} \rho\right) \mathrm{d} x \\
& \leq C\left\|\nabla u_{2}\right\|_{L^{\infty}}\|\rho\|_{L^{2}}^{2}+C\left\|\rho_{1}\right\|_{L^{\infty}}\|\nabla u\|_{L^{2}}\|\rho\|_{L^{2}}+C\|u\|_{L^{6}}\left\|\nabla \rho_{1}\right\|_{L^{3}}\|\rho\|_{L^{2}} \\
& \leq C\left\|\nabla u_{2}\right\|_{L^{\infty}}\|\rho\|_{L^{2}}^{2}+C\|\nabla u\|_{L^{2}}\|\rho\|_{L^{2}}
\end{aligned}
$$

which gives

$$
\frac{\mathrm{d}}{\mathrm{d} t}\|\rho\|_{L^{2}} \leq C\left\|\nabla u_{2}\right\|_{L^{\infty}}\|\rho\|_{L^{2}}+C\|\nabla u\|_{L^{2}}
$$

By the Gronwall inequality, we get

$$
\|\rho\|_{L^{2}} \leq C \int_{0}^{t}\|\nabla u\|_{L^{2}} \mathrm{~d} s
$$

Testing (3.2) by $u$, using (1.1a), (1.4), and (3.5), we find that

$$
\begin{aligned}
& \frac{1}{2} \frac{\mathrm{d}}{\mathrm{d} t}\left(\int \rho_{1}|u|^{2} \mathrm{~d} x+\int_{0}^{t} \int\left(\mu|\nabla u|^{2}+(\lambda+\mu)(\operatorname{div} u)^{2}\right) \mathrm{d} x \mathrm{~d} s\right) \\
& \quad+\frac{1}{2} \int\left(\mu|\nabla u|^{2}+(\lambda+\mu)(\operatorname{div} u)^{2}\right) \mathrm{d} x \\
& \leq C\left\|\nabla u_{2}\right\|_{L^{\infty}} \int \rho_{1}|u|^{2} \mathrm{~d} x+C\left\|\partial_{t} u_{2}\right\|_{L^{6}}\|u\|_{L^{6}}\|\rho\|_{L^{\frac{3}{2}}}+C\left\|u_{2}\right\|_{L^{6}}\left\|\nabla u_{2}\right\|_{L^{6}}\|u\|_{L^{6}}\|\rho\|_{L^{2}} \\
& \quad+C\left\|p\left(\rho_{1}\right)-p\left(\rho_{2}\right)\right\|_{L^{2}}\|\nabla u\|_{L^{2}}+C\left(\left\|\nabla d_{1}\right\|_{L^{\infty}}+\left\|\nabla d_{2}\right\|_{L^{\infty}}\right)\|\nabla d\|_{L^{2}}\|\nabla u\|_{L^{2}} \\
& \leq C\left\|\nabla u_{2}\right\|_{L^{\infty}} \int \rho_{1}|u|^{2} \mathrm{~d} x+C\left\|\nabla u_{2 t}\right\|_{L^{2}}\|\nabla u\|_{L^{2}}\|\rho\|_{L^{2}}+C\left\|u_{2}\right\|_{H^{2}}\|\nabla u\|_{L^{2}}\|\rho\|_{L^{2}} \\
& \quad+C\|\rho\|_{L^{2}}\|\nabla u\|_{L^{2}}+C\left(\left\|\nabla d_{1}\right\|_{L^{\infty}}+\left\|\nabla d_{2}\right\|_{L^{\infty}}\right)\|\nabla d\|_{L^{2}}\|\nabla u\|_{L^{2}} \\
& \leq C\left\|\nabla u_{2}\right\|_{L^{\infty}} \int \rho_{1}|u|^{2} \mathrm{~d} x+C\left(\left\|\nabla u_{2 t}\right\|_{L^{2}}+\|u\|_{H^{2}}+1\right)\|\nabla u\|_{L^{2}} \int_{0}^{t}\|\nabla u\|_{L^{2}} \mathrm{~d} s \\
& \quad+C\left(\left\|\nabla d_{1}\right\|_{L^{\infty}}+\left\|\nabla d_{2}\right\| \|_{L^{\infty}}\right)\|\nabla u\|_{L^{2}}\|\nabla d\|_{L^{2}} \\
& \leq \frac{\mu}{16}\|\nabla u\|_{L^{2}}^{2}+C\left\|\nabla u_{2}\right\|_{L^{\infty}} \int \rho_{1}|u|^{2} \mathrm{~d} x+C\left(\left\|\nabla u_{2 t}\right\|_{L^{2}}^{2}+\|u\|_{H^{2}}^{2}+1\right)\left(\int_{0}^{t}\|\nabla u\|_{L^{2}} \mathrm{~d} s\right)^{2} \\
& \quad+C\left(\left\|\nabla d_{1}\right\|_{L^{\infty}}^{2}+\left\|\nabla d_{2}\right\|_{L^{\infty}}^{2}\right)\|\nabla d\|_{L^{2}}^{2} \\
& \leq \frac{\mu}{16}\|\nabla u\|_{L^{2}}^{2}+C\left\|\nabla u_{2}\right\|_{L^{\infty}} \int \rho_{1}|u|^{2} \mathrm{~d} x+C\left(t\left\|\nabla u_{2 t}\right\|_{L^{2}}^{2}+\|u\|_{H^{2}}^{2}+1\right) \int_{0}^{t}\|\nabla u\|_{L^{2}}^{2} \mathrm{~d} s \\
& \quad+C\left(\left\|\nabla d_{1}\right\|_{L^{\infty}}^{2}+\left\|\nabla d_{2}\right\|_{L^{\infty}}^{2}\right)\|\nabla d\|_{L^{2}}^{2} .
\end{aligned}
$$


Testing (3.3) by $d$ and using (1.4), we have

$$
\begin{aligned}
& \quad \frac{1}{2} \frac{\mathrm{d}}{\mathrm{d} t} \int|d|^{2} \mathrm{~d} x+\int|\nabla d|^{2} \mathrm{~d} x \\
& \leq\left\|u_{1}\right\|_{L^{6}}\|\nabla d\|_{L^{2}}\|d\|_{L^{3}}+\|u\|_{L^{6}}\left\|\nabla d_{2}\right\|_{L^{3}}\|d\|_{L^{2}}+\|d\|_{L^{2}}^{2}\left\|\nabla d_{1}\right\|_{L^{\infty}}^{2} \\
& \quad+C\left(\left\|\nabla d_{1}\right\|_{L^{\infty}}+\left\|\nabla d_{2}\right\|_{L^{\infty}}\right)\|\nabla d\|_{L^{2}}\|d\|_{L^{2}} \\
& \leq C\|\nabla d\|_{L^{2}}\|d\|_{L^{3}}+C\|d\|_{L^{2}}^{2}+C\left\|\nabla d_{1}\right\|_{L^{\infty}}^{2}\|d\|_{L^{2}}^{2} \\
& \quad+C\left(\left\|\nabla d_{1}\right\|_{L^{\infty}}+\left\|\nabla d_{2}\right\|_{L^{\infty}}\right)\|\nabla d\|_{L^{2}}\|d\|_{L^{2}}+\frac{\mu}{16}\|\nabla u\|_{L^{2}}^{2} .
\end{aligned}
$$

Testing (3.3) by $-\Delta d$ and using (1.4), we have

$$
\begin{aligned}
& \quad \frac{1}{2} \frac{\mathrm{d}}{\mathrm{d} t} \int|\nabla d|^{2} \mathrm{~d} x+\int|\Delta d|^{2} \mathrm{~d} x \\
& \leq\left\|u_{1}\right\|_{L^{6}}\|\nabla d\|_{L^{3}}\|\Delta d\|_{L^{2}}+\|u\|_{L^{6}}\left\|\nabla d_{2}\right\|_{L^{3}}\|\Delta d\|_{L^{2}} \\
& \quad+C\|d\|_{L^{6}}\|\Delta d\|_{L^{2}}\left\|\nabla d_{1}\right\|_{L^{6}}^{2}+C\left(\left\|\nabla d_{1}\right\|_{L^{\infty}}+\left\|\nabla d_{2}\right\|_{L^{\infty}}\right)\|\nabla d\|_{L^{2}}\|\Delta d\|_{L^{2}} \\
& \leq C_{1} \mu\|\nabla u\|_{L^{2}}^{2}+\frac{1}{2}\|\Delta d\|_{L^{2}}^{2}+C\|\nabla d\|_{L^{2}}^{2}+C\|d\|_{L^{2}}^{2} \\
& \quad+C\left(\left\|\nabla d_{1}\right\|_{L^{\infty}}^{2}+\left\|\nabla d_{2}\right\|_{L^{\infty}}^{2}\right)\|\nabla d\|_{L^{2}}^{2} .
\end{aligned}
$$

Doing (3.6) $\times 8 C_{1}+(3.7)+(3.8)$ and using the Gronwall inequality, we arrive at

$$
\rho=0, \quad u=0 \text { and } d=0 .
$$

This completes the proof of Theorem 1.1.

\section{Acknowledgements}

Fan is supported by NSFC (Grant No. 11971234). Li is supported by NSFC (Grant No. 11671193) and A Project Funded by the Priority Academic Program Development of Jiangsu Higher Education Institutions.

\section{References}

[1] Y. Chu, X.-G. Liu and X. Liu, Strong solutions to the compressible liquid crystal system, Pac. J. Math., 257 (2012), 37-52.

[2] S. Ding, J. Huang, H. Wen and R. Zi, Incompressible limit of the compressible nematic liquid crystal flow, J. Funct. Anal., 264 (2013), 1711-1756.

[3] T. Huang, C. Wang and H. Wen, Strong solutions of the compressible nematic liquid crystal flow, J. Differential Equations, 252 (2012), 2222-2265.

[4] F. Jiang, S. Jiang and D. Wang, On multi-dimensional compressible flow of nematic liquid crystals with large initial energy in a bounded domain, J. Funct. Anal., 265 (2013), 3369-3397. 
[5] X. Li and B. Guo, Well-posedness for the three-dimensional compressible liquid crystal flows, Discrete Contin. Dyn. Syst. Ser. S, 9 (2016), 1913-1937.

[6] F. Lin and C. Wang, Recent developments of analysis for hydrodynamic flow of nematic liquid crystals, Philos. Trans. R. Soc. Lond. Ser. A Math. Phys. Eng. Sci., 372 (2014), 20130361.

[7] J. Lin, B. Lai and C. Wang, Global finite energy weak solutions to the compressible nematic liquid crystal flow in dimension three, SIAM J. Math. Anal., 47 (2015), 2952-2983.

[8] G. Metivier and S. Schochet, The incompressible limit of the non-isentropic Euler equations, Arch. Ration. Mech. Anal., 158 (2001), 61-90.

[9] G. Qi and J. Xu, The low Mach number limit for the compressible flow of liquid crystals, Appl. Math. Comput., 297 (2017), 39-49.

[10] X. Yang, Uniform well-posedness and low Mach number limit to the compressible nematic liquid flows in a bounded domain, Nonlinear Anal., 120 (2015), 118-126.

[11] L. Zeng, G. Ni and X. Ai, Low Mach number limit of global solutions to 3-D compressible nematic liquid crystal flows with Dirichlet boundary condition, Math. Methods Appl. Sci., 42 (2019), 2053-2068. 